\title{
AVALIAÇÃO DA EFICIÊNCIA DA MACRÓFITA HYMENACHNE GRUMOSA COMO ORGANISMO-TESTE, EM DOIS SISTEMAS DE TRATAMENTO DE EFLUENTES, ATRAVÉS DE ENSAIOS ECOTOXICOLÓGICOS
}

\author{
Geani Mohr ${ }^{l}$ \\ Adriana Dupont ${ }^{2}$ \\ Enio Leandro Machado ${ }^{3}$ \\ Eduardo A. Lobo $^{4}$
}

\begin{abstract}
RESUMO
O trabalho teve como objetivo avaliar a eficiência da macrófita Hymenachne grumosa como organismo-teste para remoção de poluentes utilizando Wetlands construídos (WC's) em duas estações experimentais, através de ensaios de toxicidade aguda tendo Daphnia magna Straus como bioindicador. Dois sistemas experimentais foram instalados; o primeiro (PA-1), proveniente da Estação de Tratamento de Esgoto (ETE-UNISC), entre abril a setembro de 2010, e o segundo, (PA-2) correspondente a um sistema de captação e tratamento de água em uma propriedade rural localizada em Vera Cruz, RS, entre setembro de 2013 a fevereiro de 2014. Os resultados para PA-1 indicaram que o efluente bruto apresentou uma Concentração Efetiva Inicial Mediana, CE (I)50\% 48h de 51,3 $\pm 17,2 \%$, correspondendo a uma amostra medianamente tóxica. Nos pontos P2, P3, P4 e P5 as amostras apresentaram-se pouco tóxicas. Para PA-2, os resultados indicaram que o efluente bruto apresentou uma CE (I)50\% 48h de $8,1 \pm 2,6 \%$, correspondendo a uma amostra extremamente tóxica. Nos pontos P2 e P3 as amostras enquadraram-se como altamente e pouco tóxicas, respectivamente. No P4 os efluentes não apresentaram toxicidade. Do ponto de vista da ecotoxicologia, os resultados sugerem que o uso de $H$. grumosa como componente principal nos WC's foi significativa, representando uma alternativa promissora para remover toxicidade de efluentes domésticos.
\end{abstract}

Palavras-chave: Efluente doméstico. Wetlands construídos. Macrófita Hymenachne grumosa. Ecotoxicologia.

\begin{abstract}
\footnotetext{
${ }^{1}$ Aluna do Curso de Ciências Biológicas da Universidade de Santa Cruz do Sul (UNISC)

<mohr.geani@yahoo.com.br>

${ }^{2}$ Mestre em Tecnologia Ambiental, Central Analítica da UNISC < adrianad@unisc.br >

${ }^{3}$ Professor de Departamento de Química e Física da UNISC < enio@unisc.br >

${ }^{4}$ Professor do Departamento de Biologia e Farmácia da UNISC <lobo@unisc.br>
}

The aim of the study was to assess the efficiency of the macrophyte Hymenachne grumosa as a test-organism for the removal of pollutants using constructed wetlands (CW's) in two experimental stations, through the use of acute toxicity assays having Daphnia magna as bioindicator. Two experimental systems were installed; the first (PA-1), that corresponds the Sewage Treatment Plant (STP-UNISC), between April and September of 2010, and the second, (PA-2), that corresponds to a water treatment system in small rural farm located in the municipality of Vera Cruz, RS, between September 2013 and February 2014. The results for PA-1 indicated that the raw effluent showed a Median Initial Effective Concentration, EC(I) $50 \% 48 \mathrm{~h}$ of $51,3 \pm 17,2 \%$, corresponding to a sample moderately toxic. At the sampling points P2, P3, P4 e P5, the samples showed low toxicity. The result for PA-2 indicated that the raw effluent showed an EC(I)50\% $48 \mathrm{~h}$ of $8,1 \pm 2,6 \%$, corresponding to a sample 
extremely toxic. At the sampling points $\mathrm{P} 2$ and $\mathrm{P} 3$, the samples were classified as highly toxic and low toxic, respectively. The effluent from point P4 showed no toxicity. Thus, from the Ecotoxicological point of view, the results indicated that the use of $H$. grumosa as a principal component in CW's was significant, representing a promising alternative for the removal of domestic pollutants.

Keywords: Domestic effluents. Constructed wetlands. Macrophyte Hymenachne grumosa. Ecotoxicology.

\section{INTRODUÇÃO}

O crescimento populacional vem ocasionando sérios impactos negativos sobre o meio ambiente neste século. O desenvolvimento da indústria e do comércio, bem como nos diversos ramos do meio rural e urbano, são considerados determinantes para as mudanças ambientais, principalmente devido à falta de saneamento básico. Fica evidente, portanto, a necessidade de aplicar técnicas alternativas e economicamente viáveis de remediação, principalmente em locais que geram fontes difusas de poluição, tais como unidades residenciais familiares e/ou pequenas coletividades, tanto urbanas quanto rurais, as quais não têm capacidade para suportar tecnologias que operam com certo grau de complexidade e que requerem mão de obra especializada (PHILIPPI et al., 2007).

Neste contexto, a fitorremediação é uma tecnologia que desperta grande interesse mundial, e surge como uma alternativa para o tratamento de efluentes (KHAN, 2001). Podese conceituar, de forma simplificada, que fitorremediação é o uso de plantas e microrganismos a elas associados, como técnica utilizada para a contenção, isolamento, remoção ou redução das concentrações de contaminantes em meio sólido, líquido ou gasoso (USEPA, 2000). As tendências de pesquisas em tratamento de efluentes abordam a remediação mais limpa, o reuso das águas, a recuperação de energia e nutrientes, a integração de métodos e processos e a análise do ciclo de vida. A fitorremediação através de wetlands construídos associa tais vantagens por ser um sistema de simples operação, baixo custo de implantação e manutenção, não exige mão-de-obra qualificada e com baixo consumo energético.

Por outro lado, o biomonitoramento de caráter ecotoxicológico constitui-se numa ferramenta de indiscutível importância para avaliar efeitos biológicos de corpos d'água complexos, bem como para a avaliação da eficiência de sistemas de tratamento (BRENTANO e LOBO, 2004). Segundo Zagatto e Bertoletti (2006), o biomonitoramento ecotoxicológico estuda os impactos potencialmente deletérios de substâncias ou compostos químicos que agem como poluentes sobre os organismos vivos, possibilitando a definição de padrões de qualidade da água. 
Sendo assim, o presente trabalho teve como objetivo avaliar a eficiência da macrófita Hymenachne grumosa como organismo-teste para remoção de poluentes utilizando Wetlands construídos (WC's) em duas estações experimentais, através de ensaios de toxicidade aguda tendo Daphnia magna Straus como bioindicador. Dois sistemas experimentais foram instalados; o primeiro (PA-1), proveniente da Estação de Tratamento de Esgoto (ETE-UNISC), entre abril a setembro de 2010, e o segundo, (PA-2) correspondente a um sistema de captação e tratamento de água em uma propriedade rural localizada em Vera Cruz, RS, entre setembro de 2013 a fevereiro de 2014.

\section{METODOLOGIA}

\subsection{Descrição do Processo Amostral 1 (PA-1)}

O sistema experimental foi instalado na Estação de Tratamento de Esgotos (ETE), no Campus da Universidade de Santa Cruz do Sul (UNISC), RS. De acordo com a Coordenação Ambiental da UNISC, o esgoto drenado para tratamento na ETE é originário dos mictórios e bacias sanitárias, sendo que os restaurantes possuem sistemas de tratamento com caixa separadora de óleos e graxas e posterior encaminhamento para a ETE. A Estação de Tratamento foi dimensionada para um equivalente populacional de 18.000 pessoas (estudantes e funcionários), sendo que a média por semestre é de 11.500 indivíduos divididos nos três turnos de funcionamento das aulas no campus.

Para este estudo, foram construídas quatro diferentes configurações de sistemas WC's, todas elas constituídas de três compartimentos (caixas) sequenciais precedidas por um tanque equalizador de 1.000 litros de volume útil, um decantador/digestor e um distribuidor e controlador de vazões. Todos os WC's foram dispostos sobre uma estrutura metálica na forma de degraus de escada, estando à caixa inicial de cada sistema a 1,4 m do chão; a caixa intermediária a $75 \mathrm{~cm}$ do chão e, por último, a caixa final, a $10 \mathrm{~cm}$ do chão (Fig. 1). 


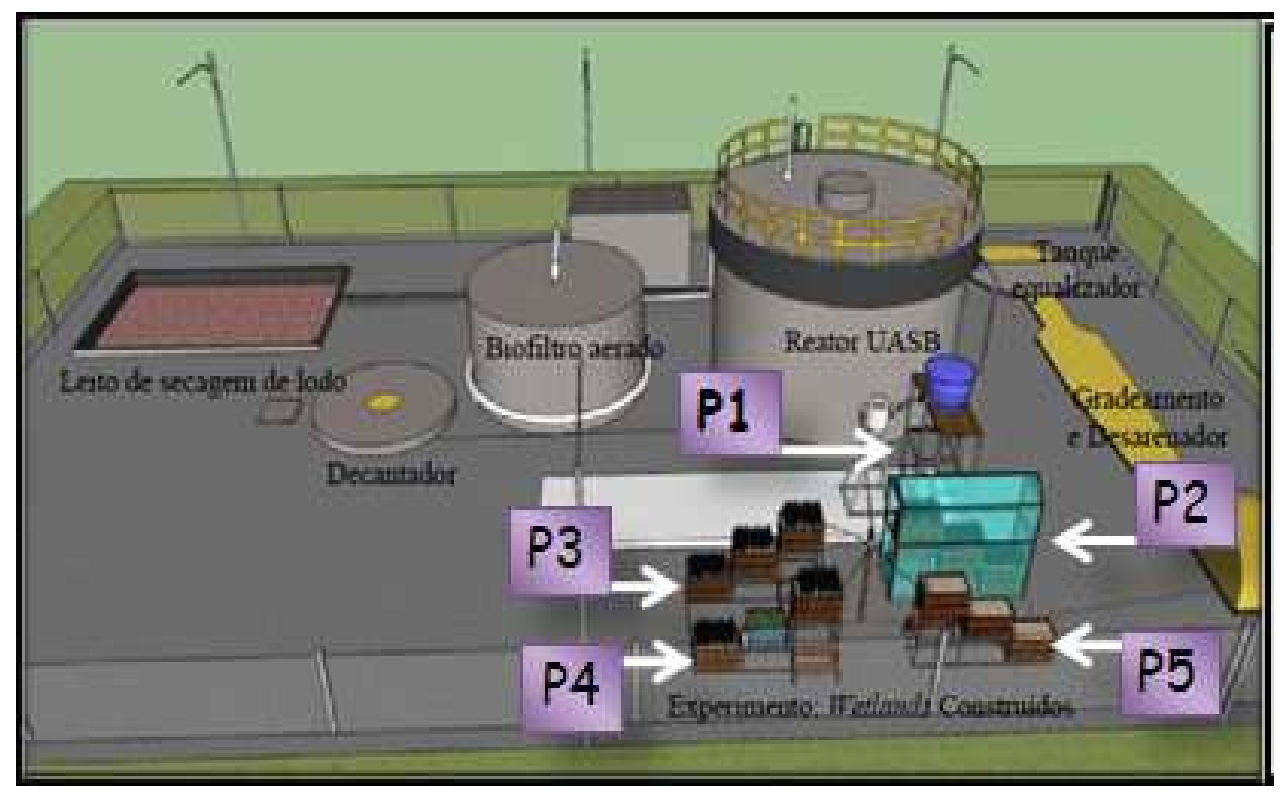

Figura 1. Unidade experimental do PA-1, Pontos de coleta, P1 (Pós reator UASB), P2 (Saída dos wetlands 1), P3 (Saída dos wetlands 2), P4 (Saída dos Wetlands 3) e P5 (Saída dos Wetlands 4).

\subsection{Metodologia de Amostragem.}

Ao todo, 35 amostras foram coletadas, entre os meses de abril a setembro de 2010, de cinco fases distintas (Pós reator UASB - P1, saída dos Wetlands 1- P2, Saída dos Wetlands 2 - P3, Saída dos Wetlands 3 - P4 e saída dos Wetlands 4 - P5).

\subsection{Descrição do Processo Amostral 2 (PA - 2)}

A propriedade rural escolhida para a instalação de um sistema de captação e tratamento de água encontra-se no município de Vera Cruz, RS, que apresenta como meio de subsistência atividades relacionadas à agricultura familiar, como a plantação de tabaco. A estação experimental de tratamento e reuso de esgoto doméstico utilizada nesta pesquisa consistiu em um sistema composto por um banheiro e uma lavanderia, dois reatores anaeróbios, quatro Wetlands construídos sequencialmente e um sistema de lâmpadas de radiação ultravioleta (UV) (Fig. 2).

Os processos empregados para o tratamento do esgoto foram: o tratamento primário (dois reatores UASB); tratamento secundário/terciário sistema do tipo Wetlands construídos (WC's) com regime de fluxo horizontal, sendo quatro leitos, com a macrófita Hymenachne grumosa (Fig. 3). O efluente gerado segue por ação da gravidade para os reatores anaeróbios e posteriormente para o sistema de WC's, sendo direcionado a uma caixa de passagem de fibra 
de vidro, com volume de 250 litros, onde, através de uma bomba é levado para o sistema de desinfecção por lâmpadas UV para somente então ser reutilizado.

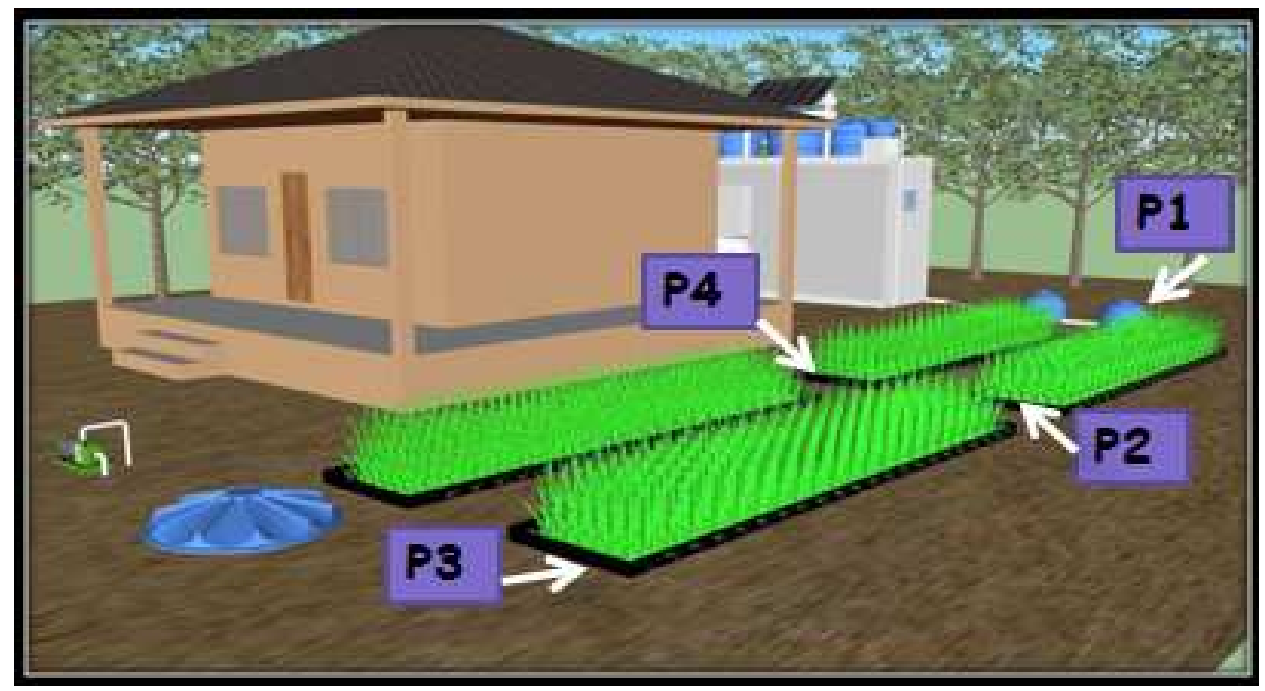

Figura 2. Maquete da Estação Experimental de tratamento - P1: Sistema Biológico - UASB; P2, P3 e P4: Wetlands construídos (Retirada de KÖHLER et al., 2012).
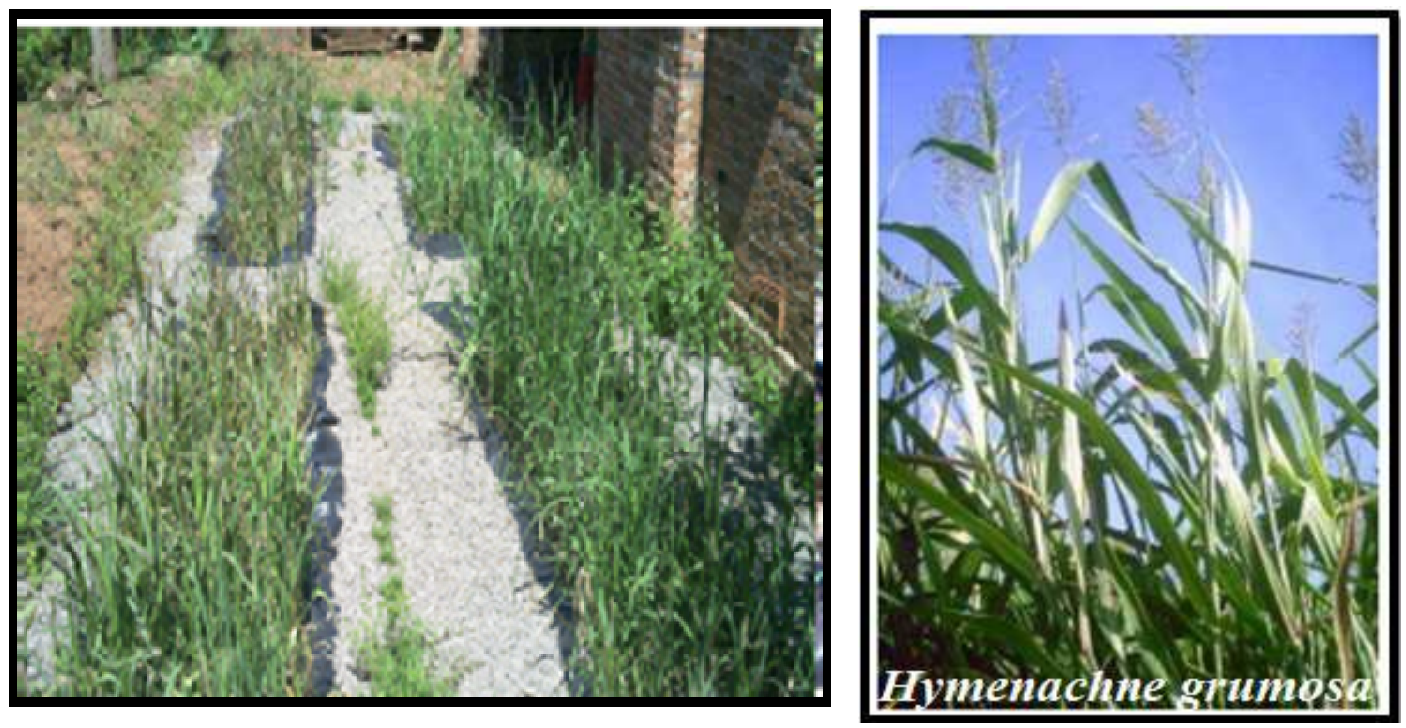

Figura 3. Estação Experimental de tratamento destacando os Wetlands construídos destacando a macrófita Hymenachne grumosa (Retirada de KÖHLER et al., 2012).

\subsection{Metodologia de Amostragem.}

Ao todo, 23 amostras foram coletadas mensalmente entre os meses de setembro de 2013 a fevereiro de 2014, de cinco fases distintas (Pós reator UASB - P1, Saída dos wetlands 1 - P2, Saída dos wetlands 2 - P3 e Saída dos Wetlands 3 - P4).

Para ambas as pesquisas realizaram-se testes ecotoxicológicos com a espécie $D$. magna, baseado na mortalidade e/ou imobilidade dos organismos testados, calculando-se a 
CE (I) $50 \%$ 48h, Concentração Efetiva Inicial Mediana, concentração da amostra no inicio do ensaio que causa efeito agudo a 50\% dos organismos em 48h, seguindo norma NBR 12713 (ABNT, 2009).

\subsection{Metodologia de Cultivo Daphnia magna e Teste Ecotoxicológico.}

A pesquisa foi desenvolvida no Laboratório de Ecotoxicologia da Universidade de Santa Cruz do Sul - UNISC. Dessa forma, os procedimentos da rotina de cultivo e testes ecotoxicológicos para Daphnia magna realizados seguiram a norma ABNT (2009). A sensibilidade dos organismos foi testada mensalmente, tendo o dicromato de potássio como substância de referência. Para ensaios de toxicidade aguda, utilizou-se como bioindicador o organismo-teste Daphnia magna (Fig. 4). Este bioindicador é um microcrustáceo planctônico, de 5,0 a 6,0 mm de comprimento, que atua como consumidor primário na cadeia alimentar aquática, alimentando-se por filtração de material orgânico particulado em suspensão.

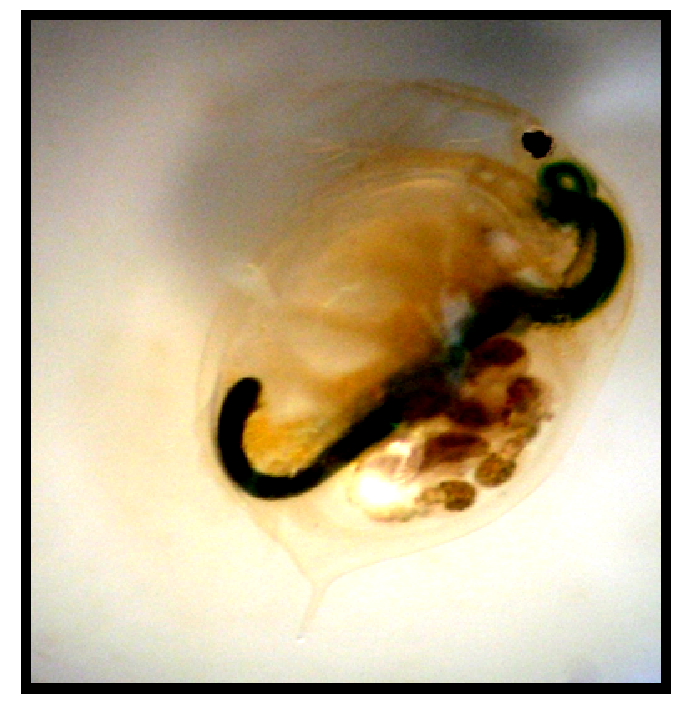

Figura 4. Daphnia magna. Aumento 40x

Os testes ecotoxicológicos foram realizados expondo os neonatos ( 2 a $26 \mathrm{~h}$ de idade) a soluções contendo o efluente. A partir da amostra, foram preparadas cinco diluições e um controle, sendo que as diluições foram realizadas com precisão volumétrica, utilizando-se progressão geométrica de razão 1/2 (Fig. 5). No controle negativo e como diluente, foi utilizado o meio ISO, também descrito na norma NBR 12713 (ABNT, 2009).

Baseando-se na imobilidade dos organismos testados foi calculada a porcentagem de imobilidade por concentrações e estimada a CE (I)50 48h, (Concentração Efetiva Inicial 
Mediana - concentração da amostra no início do ensaio que causa efeito agudo a $50 \%$ dos organismos em $48 \mathrm{~h}$, nas condições de ensaio), calculada utilizando o método estatístico Trimmed Sperman-Karber (HAMILTON, 1979) para dados não paramétricos.

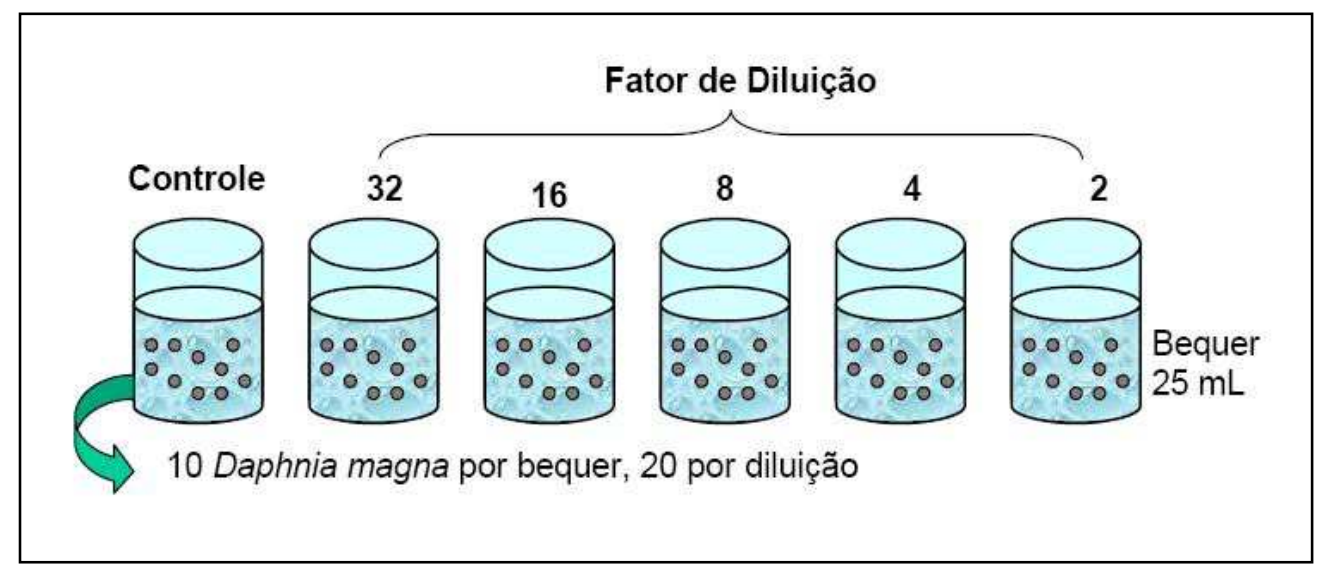

Figura 5. Análise ecotoxicológica, cinco diluições mais o controle.

Visando atribuir níveis de toxicidade às amostras testadas, foi utilizada uma escala de toxicidade relativa, conforme mostra a Tabela 1 (LOBO et al., 2006), tendo como base os resultados das determinações da CE (I)50 48h.

Tabela 1. Escala de toxicidade relativa para CE (I)50 48h (\%) com Daphnia magna.

\begin{tabular}{clc}
\hline Percentil & CE(I)50 48h & Toxicidade relativa \\
\hline $25^{\circ}$ & $<25 \%$ & Extremamente tóxica \\
$50^{\circ}$ & $25-50 \%$ & Altamente tóxica \\
$75^{\circ}$ & $50-75 \%$ & Medianamente tóxica \\
& $>75 \%$ & Pouco tóxica \\
\hline
\end{tabular}

No processamento da informação, empregou-se a estatística descritiva para a tabulação dos dados e sua ilustração gráfica, como por exemplo, gráfico de barras para a visualização e interpretação da distribuição percentual dos resultados dos testes de toxicidade (JOHNSON e BHATTACHARYYA, 1986).

\section{RESULTADOS E DISCUSSÃO}

Com relação à sensibilidade dos organismos-teste, a CE (I)50 24h média dos testes de sensibilidade ao dicromato de potássio estabeleceu-se em $0,681 \pm 0,121 \mathrm{mg} \mathrm{L}^{-1}$ 
(Coeficiente de Variação - C.V. = 17,7\%), validando assim, as condições de realização dos testes, sendo a faixa mínima aceitável de $0,560 \mathrm{mLL}^{-1}$ e máxima de $0,810 \mathrm{mgL}^{-1}$.

Os resultados para o PA-1 indicaram que o efluente bruto (P1) apresentou uma CE (I) $50 \% 48 \mathrm{~h}$ de $51,3 \pm 17,2 \%(\mathrm{CV}=33.8 \%)$, correspondendo a uma amostra medianamente tóxica. Nos pontos P2, P3, P4 e P5 as amostras apresentaram uma CE (I) 50\% 48h de 97,4\% \pm $6,8 \%(\mathrm{CV}=7 \%), 90,1 \% \pm 13,5 \%(\mathrm{CV}=15 \%), 90,6 \% \pm 16,0 \%(\mathrm{CV}=17,7 \%)$ e $82,1 \% \pm$ $16,5 \%(\mathrm{CV}=20 \%)$, correspondendo a amostras pouco tóxicas, respectivamente (Fig.5).

Para o PA-2, os resultados indicaram que o efluente bruto P1 apresentou uma CE (I) $50 \% 48 \mathrm{~h}$ de $8,1 \pm 2,6 \%(\mathrm{CV}=32 \%)$, correspondendo a uma amostra extremamente tóxica. No ponto P2, as amostras apresentaram uma CE (I)50\% 48h de 38,0\% $\pm 11,6 \%(\mathrm{CV}=$ 11,6\%), correspondendo a efluentes altamente tóxicos. As amostras do P3 apresentaram uma CE (I) $50 \%$ 48h média de 86,6\% $\pm 20,7 \%(\mathrm{CV}=23,7 \%)$, correspondendo a um efluente pouco tóxico. Entretanto, no P4, os efluentes não apresentaram toxicidade (Fig. 6).

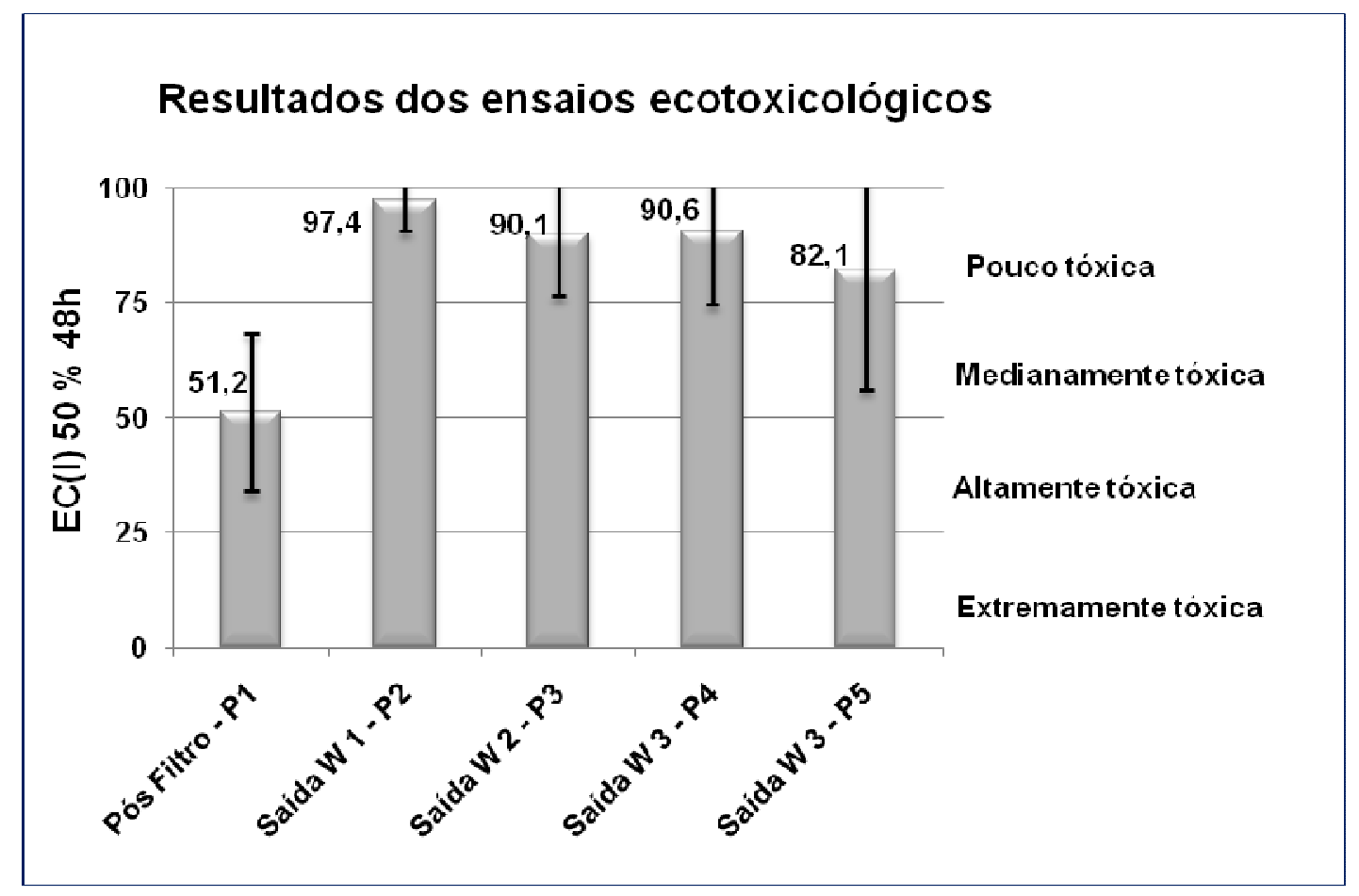

Figura 5. Resultados dos ensaios ecotoxicológicos do PA - 1. 


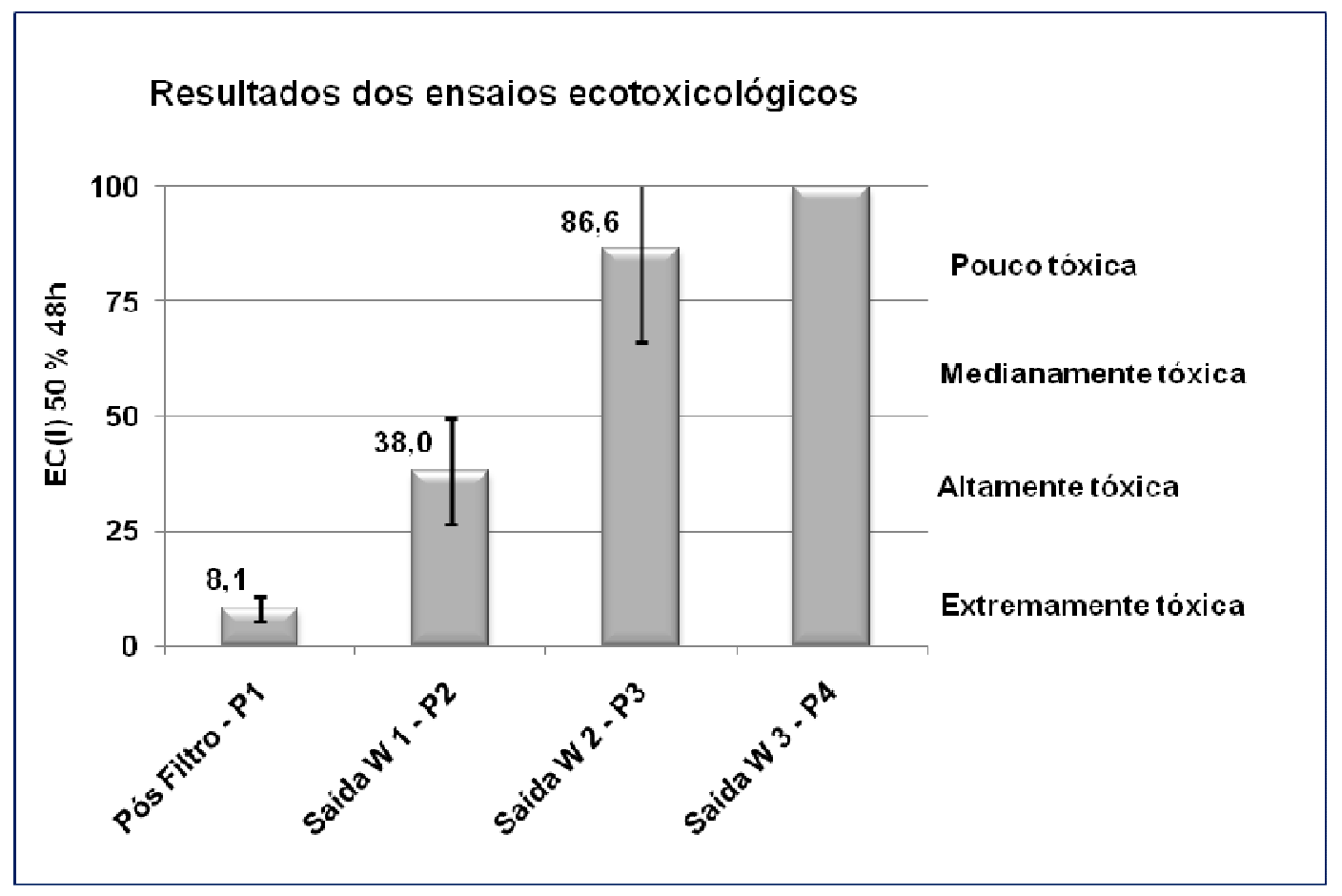

Figura 6. Resultados dos ensaios ecotoxicológicos do PA - 2.

Do ponto de vista da ecotoxicologia, os resultados indicaram que os efluentes do PA1 e do PA-2, após a saída do reator UASB (P1), foram ineficientes em relação à detoxificação, sendo classificado como medianamente tóxico e extremamente tóxico, respectivamente. Esta toxicidade é devida, provavelmente, ao elevado nível de nitrogênio amoniacal detectado no efluente, uma vez que o efluente é composto basicamente de urina. No PA-1, após passar pelos Wetlands construídos P2, P3, P4 e P5, observou-se que não ocorreu a total detoxificação do efluente, ficando na faixa de pouco tóxico.

Para o PA-2, após passar pela primeira sequência dos Wetlands construídos, observou-se que no P2 não ocorreu a total detoxificação do efluente, ficando na faixa de altamente tóxico. Entretanto, quando este efluente foi direcionado ao ponto P3, passou para a faixa de pouco tóxica, e, no P4, houve a completa detoxificação do mesmo, indicando que a integração de diferentes métodos de tratamento de efluentes destaca-se como alternativa promissora na tentativa de minimizar as ineficiências de cada tratamento.

Os Wetlands são utilizados no tratamento secundário e terciário, especialmente quando se trata de efluentes domésticos, porém torna-se necessário o tratamento primário para impedir a acumulação de sólidos, evitando assim o processo de colmatação (VAN KAICK, 2002). Segundo Souza et al. (2000), Zanella et al. (2009) e Horn (2011), o uso combinado do 
reator UASB e Wetlands construídos destacam-se como um sistema bastante eficaz para tratamento de efluentes domésticos.

Ainda assim, os resultados obtidos sugerem que a utilização da macrófita Hymenachne grumosa como componente principal nos WC's, foi altamente significativa, uma vez que houve a completa detoxificação do efluente, coincidindo com os resultados de Silveira (2010), que sugere o uso desta macrófita por possuir características desejáveis para o tratamento com Wetlands e também pelo seu excelente desenvolvimento.

Desta forma, os resultados obtidos no PA-2 sugerem que sistemas de tratamento com Wetlands construídos em fluxo vertical, leitos sequenciais e a utilização da macrófita $H$. grumosa como componente principal, foi altamente significativa, representando uma alternativa promissora para a remoção de toxicidade de efluentes domésticos.

\section{REFERÊNCIAS}

ABNT - ASSOCIAÇÃO BRASILEIRA DE NORMAS TÉCNICAS. NBR 12713: Ecotoxicologia aquática - Toxicidade aguda - Método de ensaio com Daphnia spp. (Cladócera, Crustácea). 3. ed. São Paulo, 2009.

BRENTANO, D. M., LOBO, E. A. Avaliação ecotoxicológica no processo produtivo de um curtume, utilizando Daphnia magna Straus como organismo-teste. Revista Brasileira de toxicologia, São Paulo, n. 17, v. 2, p. 13-18. dez. 2004.

HAMILTON, M. A., RUSSO, R. C., THURSTON, R. V. Trimmed Spearmann-Karber metod for calculation of EC50 and LC values in bioassays. Burlington Research, n. 11, v. 7, p. 114119, 1979.

HORN, T. B. Integração de sistemas Wetlands construídos + fotoozonização catalítica no tratamento de efluentes de campus universitário. Santa Cruz do Sul, 2011. 157 p. Dissertação (Mestrado), Programa de Pós - Graduação em Tecnologia Ambiental, Universidade de Santa Cruz do Sul, 2011.

JOHNSON, R. A., BHATTACHARYYA, G. K. Statistics: Principles and Methods. New York: John Wiley \& Sons, 1986.

KHAN, A. G. Relationships between chromium biomagnification ratio, accumulation factor, and mycorrhizae in plants growing on tannery effluent-polluted soil. Environmental International [online], n. 5-6, v. 26, p. 417-423, maio 2001.

KÖHLER, A., MACHADO, E. L., ZENWES, F. V. Sistema de captação e tratamento de águas em pequenas propriedades visando sua reutilização. Santa Cruz do Sul: Lupagraf, 2012. 12 p.

LOBO, E. A., RATHKE, F. S., BRENTANO, D. M. Ecotoxicologia aplicada: o caso dos produtores de tabaco na bacia hidrográfica do Rio Pardinho, RS, Brasil. In: ETGES, V. E., 
FERREIRA, M. A. F. A produção do tabaco: impacto no ecossistema e na saúde humana na região de Santa Cruz do Sul, RS. Santa Cruz do Sul: EDUNISC, 2006. p. 41-68.

PHILIPPI, L. S., SEZERINO, P. H., CAMPOS, R. H. Sistemas descentralizados de tratamento de esgotos. Florianópolis: Pandion, 2007.63 p.

SILVEIRA, D. Estudos fenológicos da macrófita Hymenachne grumosa (Magnoliophyta Poaceae) na aplicação de Wetlands construídos para tratamento de efluentes secundários de campus universitário. Santa Cruz do Sul, 2010. 103 p. Dissertação (Mestrado), Programa de Pós Graduação em Tecnologia Ambiental, Universidade de Santa Cruz do Sul, 2010.

SOUZA, J. T., VAN HAANDEL, A. C., COSENTINO, P. R. S., GUIMARÃES, A. V. A. Pós-Tratamento de efluente de reator UASB utilizando sistemas "wetlands" construídos. Revista Brasileira de Engenharia Agrícola e Ambiental, Campina Grande, n. 1, v. 4, p. 87-91, jan./abr. 2000.

USEPA - UNITED STATES ENVIRONMENTAL PROTECTION AGENCY. Constructed wetlands treatment of municipal wastewater. National Risk management Research Laboratory. Office of Research and Development. Ohio: Cincinati, 2000. 166 p.

VAN KAICK, T. S. Estação de tratamento de esgoto por meio de zona de raízes: uma proposta de tecnologia apropriada para saneamento básico no litoral do Paraná. Curitiba, 2002. Dissertação (Mestrado), Programa de Pós-Graduação em Tecnologia, Centro Federal de Educação Tecnológica do Paraná, 2002.

ZAGATTO, P. A., BERTOLETTI, E. (Orgs.). Ecotoxicologia aquática - princípios e aplicações. São Carlos: Rima, 2006. 478 p.

ZANELlA, L., NOUR, E. A., ROSTON, D. M. Cyperuspapyrus em sistema de wetland construído como pós - tratamento de esgotos. ABES - Associação Brasileira de Engenharia Sanitária e Ambiental. Anais... 25 Congresso Brasileiro de Engenharia Sanitária e Ambiental. p. 10, Recife. 2009. 